\title{
Amputações de membros inferiores por Diabetes Mellitus nos estados e nas regiões do
}

\section{Brasil}

Amputation lower limb due to Diabetes Mellitus the states and regions of Brazil

Amputaciones de membros inferiores por Diabetes Mellitus em los estados y regiones de Brasil

Título em espanhol e alinhado à esquerda

Recebido: 12/03/2021 | Revisado: 19/03/2021 | Aceito: 25/03/2021 | Publicado: 02/04/2021

\author{
Ana Amancio Santos da Silva \\ ORCID: https://orcid.org/0000-0001-6966-9528 \\ Universidade Federal de Alagoas, Brasil \\ E-mail: ana.amanciophysio@gmail.com \\ Aldemar Araujo Castro \\ ORCID: https://orcid.org/0000-0003-0212-5950 \\ Universidade Estadual de Ciências da Saúde de Alagoas, Brasil \\ E-mail: aldemararaujocastro@gmail.com \\ Larissa Gabrielle De Bomfim \\ ORCID: https://orcid.org/0000-0002-5091-0577 \\ Centro Universitário Cesmac, Brasil \\ E-mail: larissa12souzab@gmail.com \\ Guilherme Benjamin Brandão Pitta \\ ORCID: https://orcid.org/0000-0003-2790-2015 \\ Universidade Federal de Alagoas, Brasil \\ E-mail: guilhermebbpitta@gmail.com
}

\begin{abstract}
Resumo
A amputação por Diabetes Mellitus consiste em uma gravidade com dimensão mundial, ocorre frequentemente em populações de baixo nível socioeconômico, em condições inadequadas de higiene e falta de acesso às unidades de saúde. O pé diabético está associado a fatores como a neuropatia diabética, doença arterial periférica e infecção, contribuindo para um prognóstico de ulceração e/ou necrose no dedo, parte do pé, pé por completo e/ou perna, amputação e morte. O objetivo deste estudo foi determinar o perfil epidemiológico da amputação em membros inferiores por diabetes mellitus nas regiões e estados do Brasil. A pesquisa foi realizada através das bases de dados do Registro de procedimentos de amputações de membros inferiores no Sistema de Informações Hospitalares (SIH/SUS) referentes ao período de 2000 a 2020. A amputação foi mais prevalente respectivamente nas regiões sudeste, nordeste, sul, centrooeste e norte. Os achados desse estudo também indicam a necessidade de promoção precoce de rastreamento, diagnóstico, tratamento, reabilitação, a fim de potencializar a cicatrização e retorno às atividades de vida diária e profissionais, evitando amputação e morte.
\end{abstract}

Palavras-chave: Amputação; Extremidade inferior; Pé diabético; Sistema de informação hospitalar.

\begin{abstract}
Amputation due to Diabetes Mellitus consists of a worldwide dimension of severity, frequently occurring in populations of low socioeconomic status in inadequate conditions of hygiene and lack of access to health facilities. The diabetic foot is associated with factors such as diabetic neuropathy, peripheral arterial disease and infection, contributing to a prognosis of ulceration and / or necrosis in the finger, part of the foot, complete foot and / or leg, amputation and death. The aim of this study was to determine the epidemiological profile of amputation in lower limbs due to diabetes mellitus in the regions and states of Brazil. The research was carried out through the databases of the Registry of lower limb amputation procedures in the Hospital Information System (SIH / SUS) for the period from 2000 to 2020. Amputation was more prevalent in the southeast, northeast, south, respectively. midwest and north. The findings of this study also indicate the need for early promotion of screening, diagnosis, treatment, rehabilitation, in order to enhance healing and return to activities of daily and professional life, avoiding amputation and death.
\end{abstract}

Keywords: Amputation; Lower Extremity; Diabetic foot; Hospital information system.

\section{Resumen}

La amputación por diabetes mellitus consiste en una dimensión mundial de la gravedad, que ocurre con frecuencia en poblaciones de bajo nivel socioeconómico en condiciones de higiene inadecuadas y falta de acceso a los servicios de salud. El pie diabético se asocia a factores como neuropatía diabética, enfermedad arterial periférica e infección, contribuyendo a un pronóstico de ulceración y / o necrosis en el dedo, parte del pie, pie y / o pierna completos, amputación y muerte. El objetivo de este estudio fue determinar el perfil epidemiológico de la amputación de miembros 
inferiores por diabetes mellitus en las regiones y estados de Brasil. La investigación se realizó a través de las bases de datos del Registro de procedimientos de amputación de miembros inferiores del Sistema de Información Hospitalaria (SIH / SUS) para el período 2000 a 2020. La amputación fue más prevalente en el sureste, noreste y sur, respectivamente. medio oeste y norte. Los hallazgos de este estudio también indican la necesidad de una promoción temprana del cribado, el diagnóstico, el tratamiento, la rehabilitación, con el fin de mejorar la curación y el regreso a las actividades de la vida diaria y profesional, evitando la amputación y la muerte.

Palabras clave: Amputación; Extremidad inferior; Pie diabético; Sistema de información em hospital.

\section{Introduçãa}

A amputação por Diabetes Mellitus ocorre mundialmente, frequentemente em pessoas com baixo nível socioeconômico, associados a condições inadequadas de higiene e falta de acesso às unidades de saúde (Assumpção et al., 2009). A gravidade clínica da doença (iniciada por ferida no pé que não cicatriza e, eventualmente resultam em perda do dedo do pé, parte do pé, do pé ou perna) e o tempo que o paciente busca assistência terapêutica, seja pela falta de acessibilidade ao serviço de saúde ou porque ele não buscou essa assistência por medo ou por desconhecimento das repercussões clínicas, nos serviços de atenção especializada esses pacientes são admitidos com lesões infecciosas e necróticas que requerem abordagem cirúrgica, como amputação, com elevado risco de morbidade e mortalidade (Geiss, et al, 2018). A mutilação, seja ela menor ou maior, provoca alterações biopsicossociais, causando sofrimento, afastamento das atividades de trabalho, aposentadoria precoce, perda de função e elevados custos para tratamento e reabilitação (Santos et al.,2006; Pitta et al., 2005; Miyajima et al., 2006).

As úlceras nos pés de pacientes diabéticos (UPD), configuram a Síndrome do Pé Diabético, que são complicações frequentes do DM, 15 a 25\% das pessoas com DM podem apresentar lesões nos pés ao longo de suas vidas (Boulton, et al., 2005). As amputações de membros inferiores (MMII) são um evento sentinela, pois o risco é influenciado pelo controle de diversos fatores (controle glicêmico, controle pressórico, tabagismo).

O DM predispõe a neuropatia diabética e doença arterial periférica, contribuindo para a perda de sensibilidade (protetora, dor e vibração) e isquemia (FDI, 2020). Traumas pequenos (sapatos mal ajustados e/ou uma lesão mecânica e/ou térmica aguda) podem precipitar essas predisposições e causar infecção e ulceração do pé. A perda da sensação de proteção, deformidades do pé e mobilidade articular limitada podem resultar em carga biomecânica anormal do pé, culminando em alto estresse mecânico, cuja resposta geralmente é o espessamento da pele (calo), levando a uma sobrecarga do pé e geralmente com hemorragia subcutânea e, eventualmente, ulceração da pele (Schaper, 2020).

A Federação Internacional de Diabetes estima que o Brasil tenha em torno de 16,8 milhões de pessoas portadoras de DM (FDI, 2020). Se as tendências atuais persistirem, estima-se que o número de diabéticos seja superior a 628,6 milhões em 2045. No Brasil, existe uma incidência crescente de DM, que coloca o país entre os quatro primeiros em relação à prevalência dessa doença a nível mundial (Atlas, I. D. 2013). As UPD precedem 85\% das amputações (Pecoraro, et al., 1990) e a DM é a principal causa de amputação de MMII. Apesar de intervenções positivas, as taxas de amputações não traumáticas entre indivíduos com DM são 10 a 20 vezes mais altas em relação aos não diabéticos (Wrobel et al., 2001).

De acordo Nunes et al., (2006), para diminuir o número de amputações, é preciso conhecer detalhadamente as necessidades de saúde dos pacientes e a forma como estão sendo cuidados, a fim de criar um plano de cuidado eficiente, o qual permita a boa evolução dos pacientes.

Nesse sentido, este trabalho tem o objetivo de determinar um perfil epidemiológico de amputação por Diabetes Mellitus nas regiões e estados do Brasil.

\section{Metodologia}


Estudo epidemiológico, descritivo, com análise quantitativa (Pereira A.S. et al. (2018) referente as amputações de membros inferiores em paciente com Diabetes Mellitus, realizado através de consulta ao banco de dados do Departamento de Informática do SUS, do Ministério da Saúde - DATASUS, o acesso a esses dados foi pela plataforma online TABNET, a partir das autorizações de internação hospitalar (AIH) aprovadas por unidade da Federação segundo ano de processamento, no Sistema de Informação Hospitalar (SIH/SUS) seleção do período de janeiro de 2000 a agosto de 2020 (DATASUS, 2021). Epidemiologia e morbidade (Janeiro de 2002 a Janeiro de 2012): Hipertensão e Diabetes (HIPERDIA), vale salientar que esses dados só estão disponíveis até abril de 2013. Os dados obtidos pelo TABNET/DATASUS foram tabulados e analisados no Excel versão 2019. Não houve submissão ao Comitê de Ética em Pesquisa, devido os dados estarem em domínio público. Foram incluídos nesta pesquisa todos os dados notificados pelos sistemas relatados anteriormente e os critérios de exclusão foram os dados que não relacionados a temática desta pesquisa.

Para avaliar a elegibilidade dos dados, utilizou-se o método PICO (P = Número de amputações; $\mathrm{I}=$ Prevalência de amputação; $\mathrm{C}$ = não tem; $\mathrm{O}=$ Amputação por regiões e estado) para melhor delineamento dos critérios específicos de inclusão e exclusão.

Variável primária

Amputações de membros inferiores por Diabetes Mellitus no Brasil

Variáveis secundárias

Amputações de membros inferiores no Brasil

Critério de inclusão

Registro de procedimentos de amputações de membros inferiores no Sistema de Informações Hospitalares (SIH/SUS)

Dados complementares:

a) Frequência por sexo

b) Frequência por idade

c) Frequência por faixa etária

Método Estatístico:

a) Hipóteses:

$\mathrm{H} 0=\mathrm{H} 1 ; \mathrm{H} 0$ \# H1 . A prevalência das amputações de membros inferiores nos estados e nas regiões do Brasil é de 20 amputações por 100 mil habitantes por ano.

\section{Resultados}

No período de 2010 a 2020, foram registrados um total de 1.510 .733 hospitalizações relacionadas ao Diabetes Mellitus, sendo as regiões que apresentaram um maior números de casos respectivamente sudeste (530.425), nordeste (484.103), sul ( 243.710), norte (137.44) e centro-oeste ( 115.054) (Gráfico 1). 
Gráfico 1 - Morbidade Hospitalar por DM no Brasil no período de 2010-2020.

\section{Morbidade Hospitalar por Diabetes Mellitus no Brasil no período de 2010 - 2020}

\subsection{0 .000}

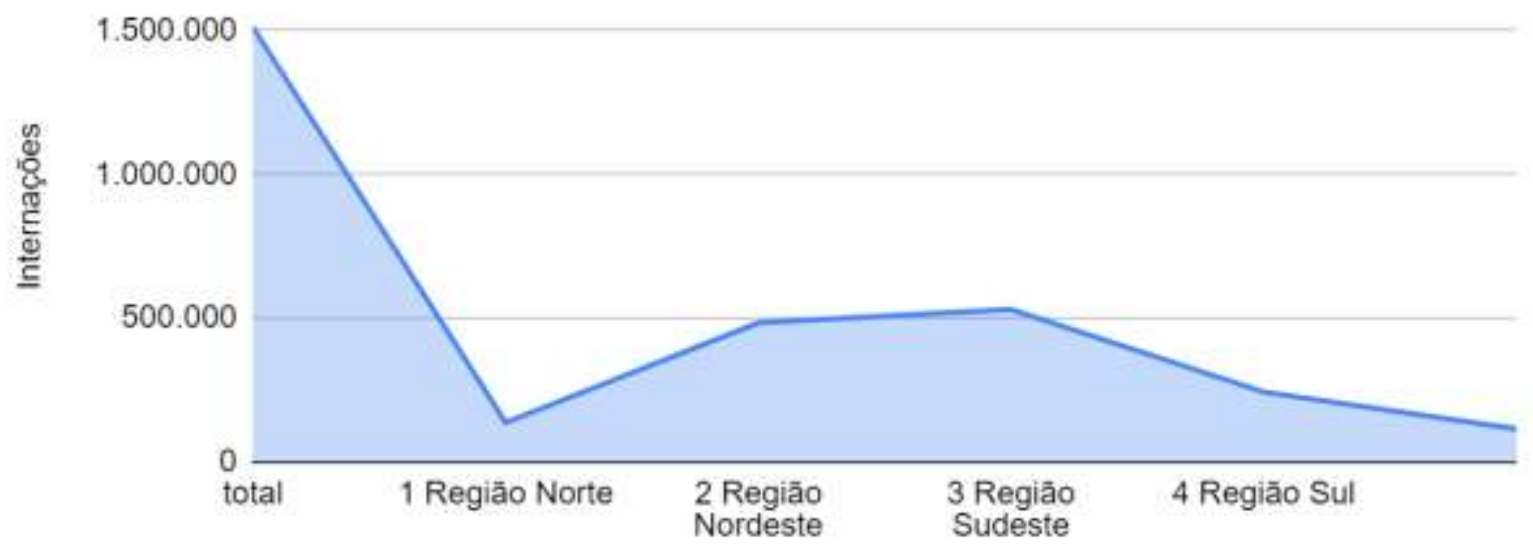

Regiāo

Fonte: Dados da pesquisa (2021).

No período de 2008 a 2017, foram registrados na 10a região de saúde de Alagoas um percentual elevado em relação ao Brasil de Diabéticos que realizaram amputação não traumática de membros inferiores (Gráfico 2).

Gráfico 2 - Percentual de usuários internados com DM que realizaram amputação não traumática de MMII

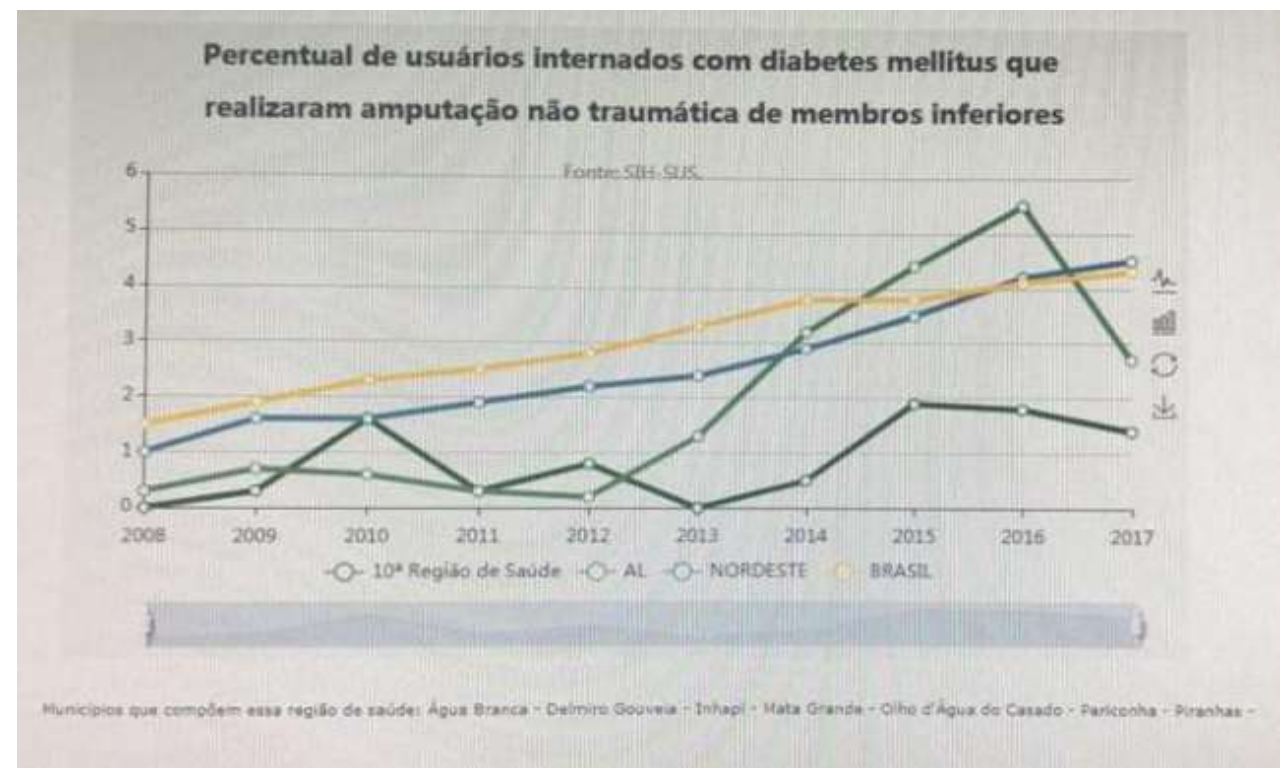

Fonte: Dados da pesquisa (2021)

No período de 2009 a 2019, foram registrados um total de 653.865 mortalidade relacionadas ao Diabetes Mellitus, sendo as regiões que apresentaram um maior números de casos respectivamente sudeste (255.390), nordeste (214.553), sul (101.121), norte (43.417) e centro-oeste (39.384) (Gráfico 3). 
Gráfico 3 - MORTALIDADE - BRASIL por Diabetes mellitus no período de 2009 - 2019.

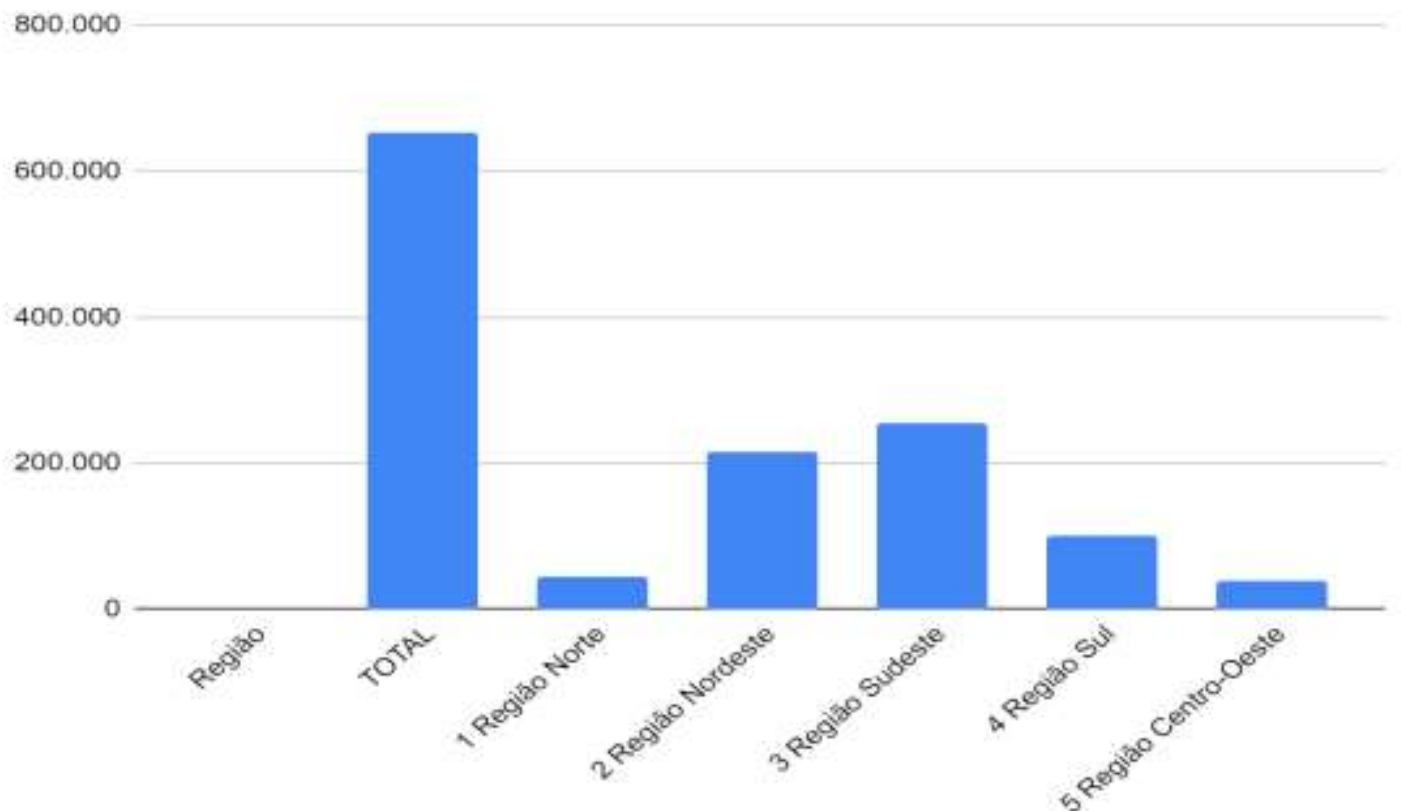

Fonte: Dados da pesquisa (2021)

No período de 2010 a 2020, foram registrados um total de 247.047 internações relacionadas a Amputação/ desarticulação de membros inferiores, sendo as regiões que apresentaram um maior números de casos respectivamente sudeste (103.908), nordeste (80.082), sul (36.013), norte (13.467) e centro-oeste (13.577) (Gráfico 4).

Gráfico 4 - No de internações por amputação/desarticulação de MMII por região brasileira entre 2010-2020.

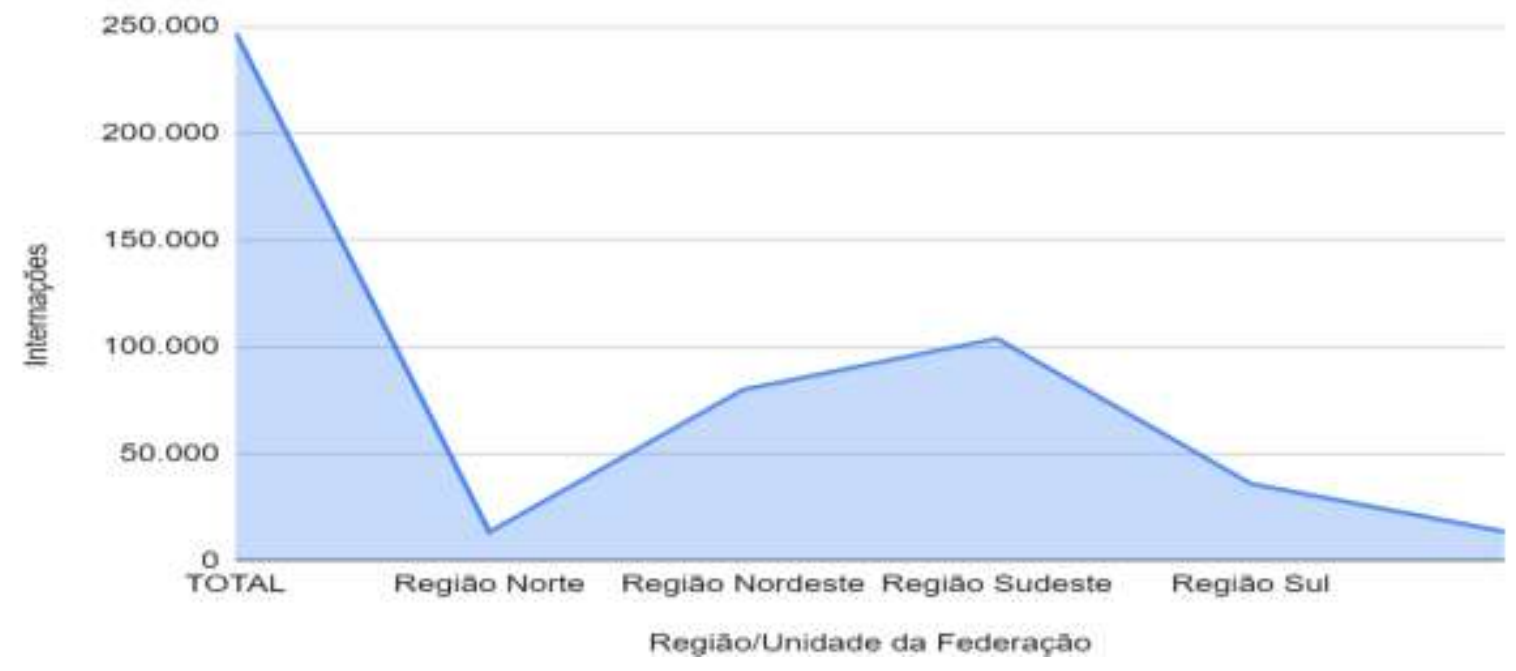

Fonte: Dados da pesquisa (2021)

No período de 2010 a 2020, foram registrados um total de 80.082 internações relacionadas a Amputação/ desarticulação de membros inferiores, sendo os estados da região nordeste que apresentaram um maior números de casos respectivamente Bahia (20.165), Pernambuco (17.325), Ceará (10.036), Maranhão (7.420), Paraíba (6.687), Alagoas (5.183), Piauí (4.941), Rio Grande do Norte (4.730) e Sergipe (3.581) (Gráfico 5). 
Research, Society and Development, v. 10, n. 4, e11910413837, 2021

(CC BY 4.0) | ISSN 2525-3409 | DOI: http://dx.doi.org/10.33448/rsd-v10i4.13837

Gráfico 5 - № de internações por amputação/desarticulação de membros inferiores entre 2010 e 2020 na região Nordeste e seus estados.

100.000

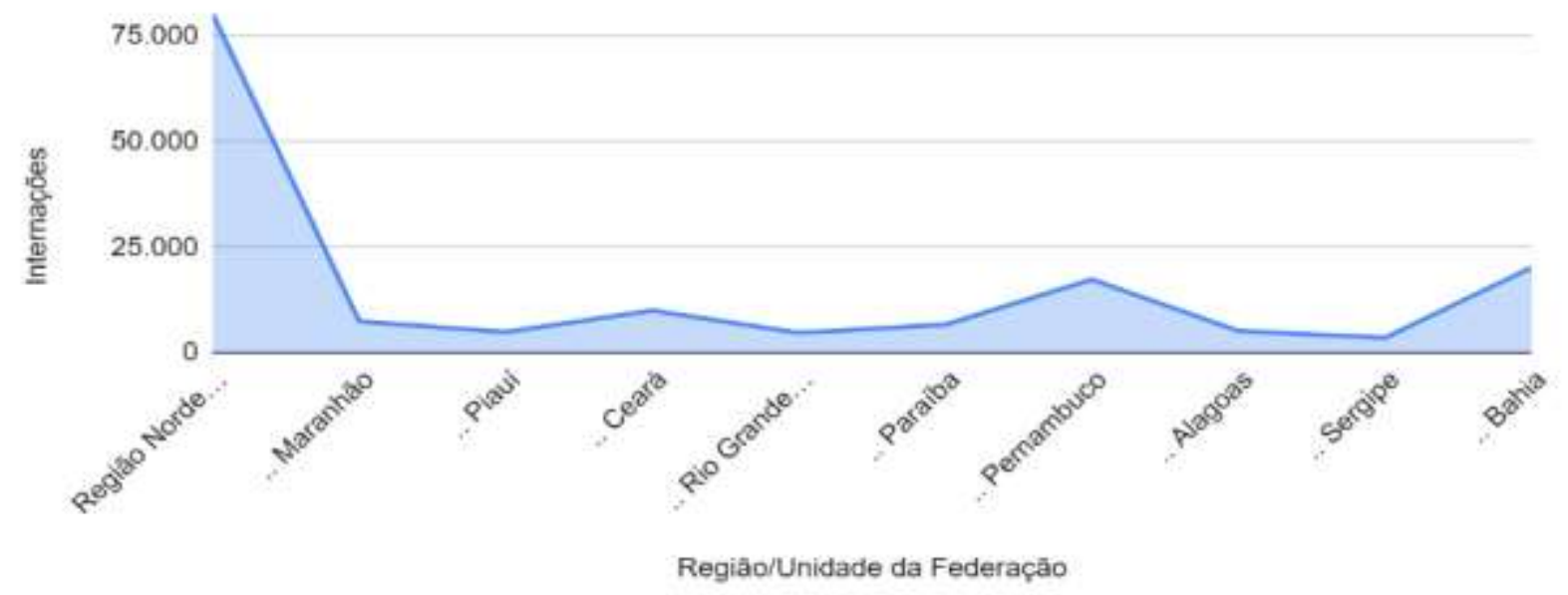

Fonte: Dados da pesquisa (2021)

No período de janeiro de 2010 a agosto de 2020, os 10 estados mais prevalentes em amputações de MMII para 100.000 habitantes são, em ordem decrescente: Piauí, Sergipe, Rio Grande do Sul, Alagoas, Pernambuco, Rio de Janeiro, Minas Gerais, Paraíba, Espírito Santo, Santa Catarina. Todos eles têm uma média de prevalência maior que essa taxa brasileira para o mesmo período (Gráfico 6). 
Gráfico 6 - Estados brasileiros mais prevalentes em amputações em MMII.

\section{MÉDIA DAS PREVALÊNCIAS}

40

\section{0 estados mais prevalentes}

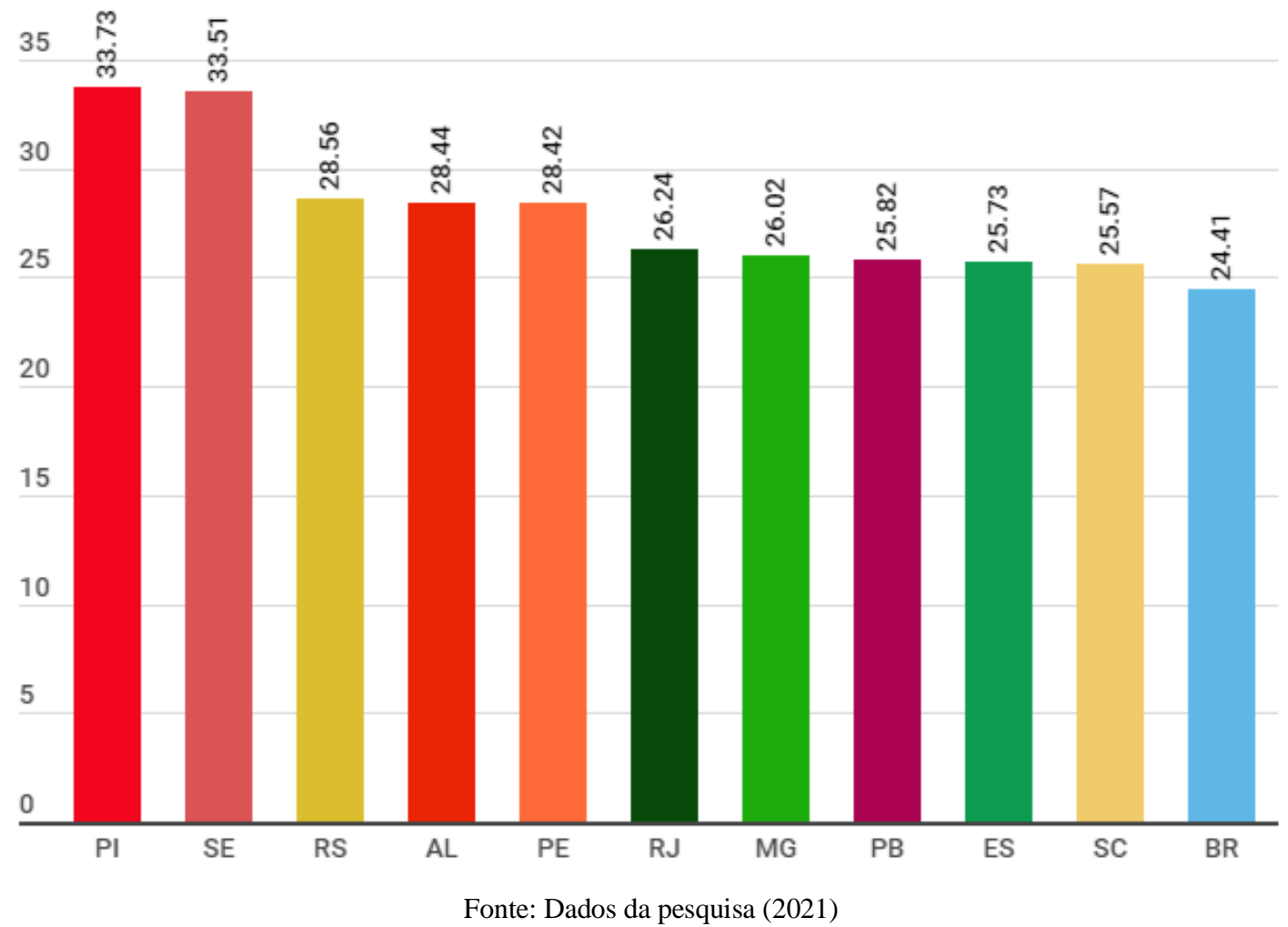

Dentre os estados da região Nordeste, as prevalências de amputações de MMII para 100.000 habitantes é bem variada tanto entre eles quanto no próprio estado ao longo dos anos. Dessa forma, vê-se que Alagoas é o estado que mais variou entre janeiro de 2010 e agosto de 2020, com um desvio padrão muito maior que a região a qual faz parte e até o Brasil (Tabela 1).

Tabela 1 - Prevalência das amputações de MMII para 100.000 habitantes nos estados brasileiros e respectivos desvios padrão.

\begin{tabular}{|c|c|c|c|c|c|c|c|c|c|c|c|}
\hline & MA & PI & CE & RN & PB & PE & AL & SE & BA & BRASIL & Nordeste \\
\hline 2010 & 16,17 & 23,08 & 17,57 & 20,87 & 26,71 & 25,49 & 14,71 & 30,78 & 19,51 & 21,09 & 20,74 \\
\hline 2011 & 20,66 & 21,26 & 21,23 & 23,54 & 26,66 & 29,70 & 9,62 & 29,20 & 21,81 & 21,85 & 22,87 \\
\hline 2012 & 19,11 & 28,58 & 18,33 & 19,74 & 23,89 & 27,33 & 9,72 & 30,85 & 23,27 & 21,67 & 22,28 \\
\hline 2013 & 20,33 & 27,85 & 18,28 & 21,48 & 26,81 & 29,62 & 17,86 & 35,61 & 21,52 & 22,62 & 23,28 \\
\hline 2014 & 25,12 & 32,18 & 24,09 & 21,47 & 25,17 & 29,66 & 26,41 & 25,10 & 22,30 & 23,55 & 25,27 \\
\hline 2015 & 27,44 & 37.25 & 27,10 & 25,08 & 25,35 & 26,28 & 32,90 & 30,50 & 25,53 & 24,38 & 27,41 \\
\hline 2016 & 26,48 & 39,40 & 25,48 & 24,37 & 23,98 & 30,29 & 41,11 & 39,87 & 24,26 & 25,35 & 28,21 \\
\hline 2017 & 27,34 & 39,88 & 26,27 & 22,49 & 25,34 & 29,48 & 44,69 & 31,72 & 25,14 & 26,06 & 28,43 \\
\hline 2018 & 29,23 & 41,52 & 30,06 & 27,31 & 25,50 & 27,84 & 43,52 & 41,48 & 30,35 & 27,98 & 31,07 \\
\hline 2019 & 25,22 & 46,33 & 32,39 & 31,54 & 28,77 & 28,51 & 43,84 & 40,02 & 30,95 & 29,55 & 31,94 \\
\hline 2020 & 15,45 & 23,29 & 22,76 & 20,15 & 18,57 & 19,96 & 7,76 & 27,69 & 24,81 & 18,93 & 20,81 \\
\hline $\begin{array}{l}\text { Desvio } \\
\text { Padrăo }\end{array}$ & 4,799 & 8,562 & 4,893 & 3,519 & 2,589 & 2,981 & 15,190 & 5,456 & 3,532 & 3,148 & 3,984 \\
\hline
\end{tabular}


Entre Janeiro de 2010 e Agosto de 2020, a região Sul e Nordeste do Brasil foram as regiões em que as prevalências de amputações de MMII para 100.000 habitantes foram as mais altas. Junto com o Sudeste, essas 3 regiões superaram o índice de amputações brasileiro para o período (Gráfico 7).

Gráfico 7 - Amputações por 100.000 habitantes entre as regiões brasileiras de 2010 a 2020.

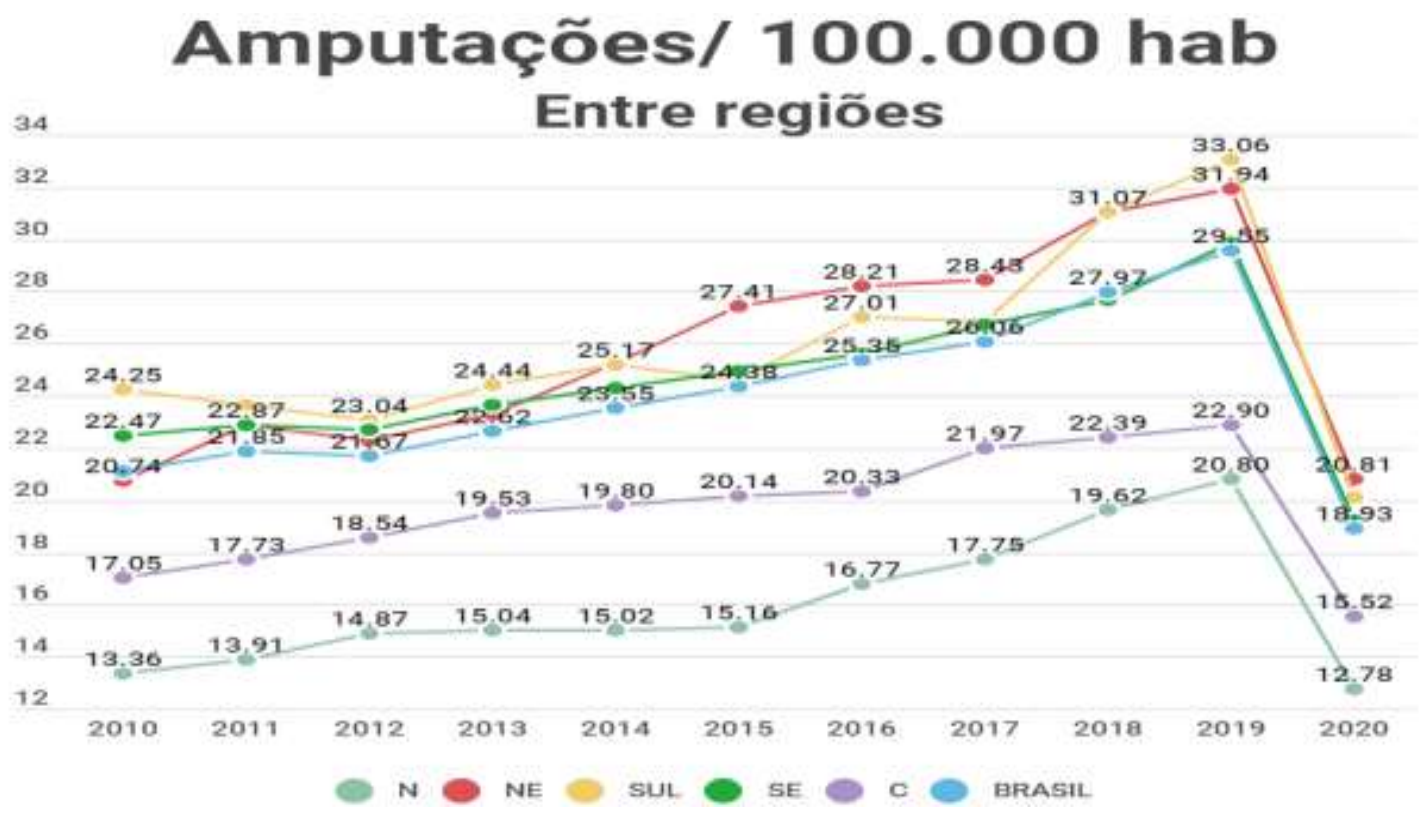

Fonte: Autoras.

Na região Nordeste, entre os anos de 2010 e 2020, Paraíba e Piauí lideraram as prevalências de amputações de MMII para 100.000 habitantes, e Alagoas foi o estado que mais variou nesse quesito, provavelmente por uma subnotificação nos anos de 2010 a 2012. A queda da prevalência em cada estado no ano de 2020 se deu pelo período de coleta de dados, pois o Sistema de Informações Hospitalares do SUS apenas constata até Agosto de 2020 (Gráfico 8). 
Gráfico 8 - Amputações por 100.000 habitantes nos estados do Nordeste entre 2010 e 2020.

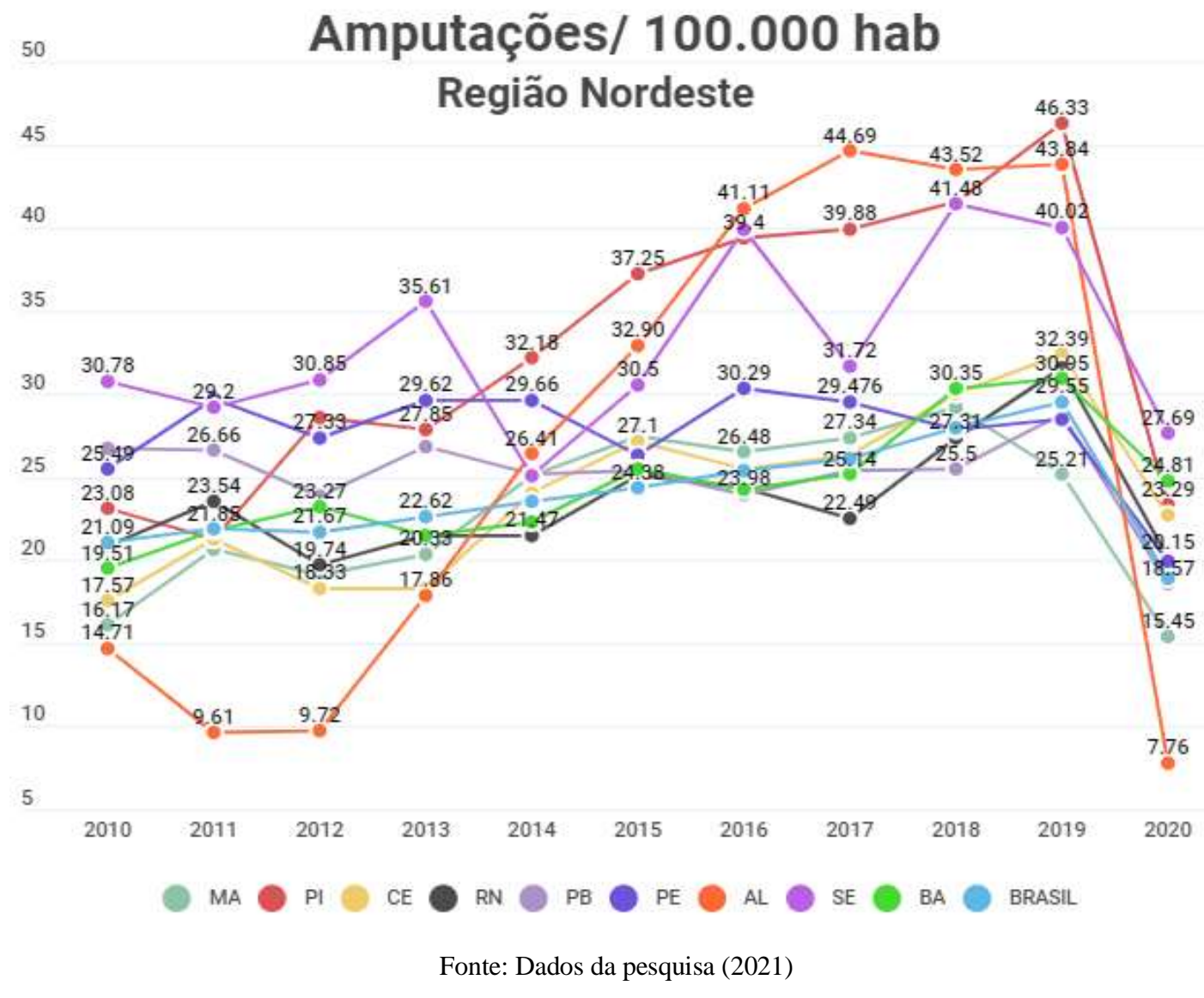

No Estado de Alagoas, entre os anos de 2002 e 2012, os municípios que lideraram amputações em pé diabético por Diabetes Mellitus de acordo com o cadastro do programa HIPERDIA foram respectivamente Maceió, Arapiraca, Palmeira dos Índios e Penedo. (Gráfico 9)

Gráfico 9 - Amputações por Diabetes Mellitus em Alagoas de acordo com o cadastro do programa HIPERDIA no período de 2002 a 2012.

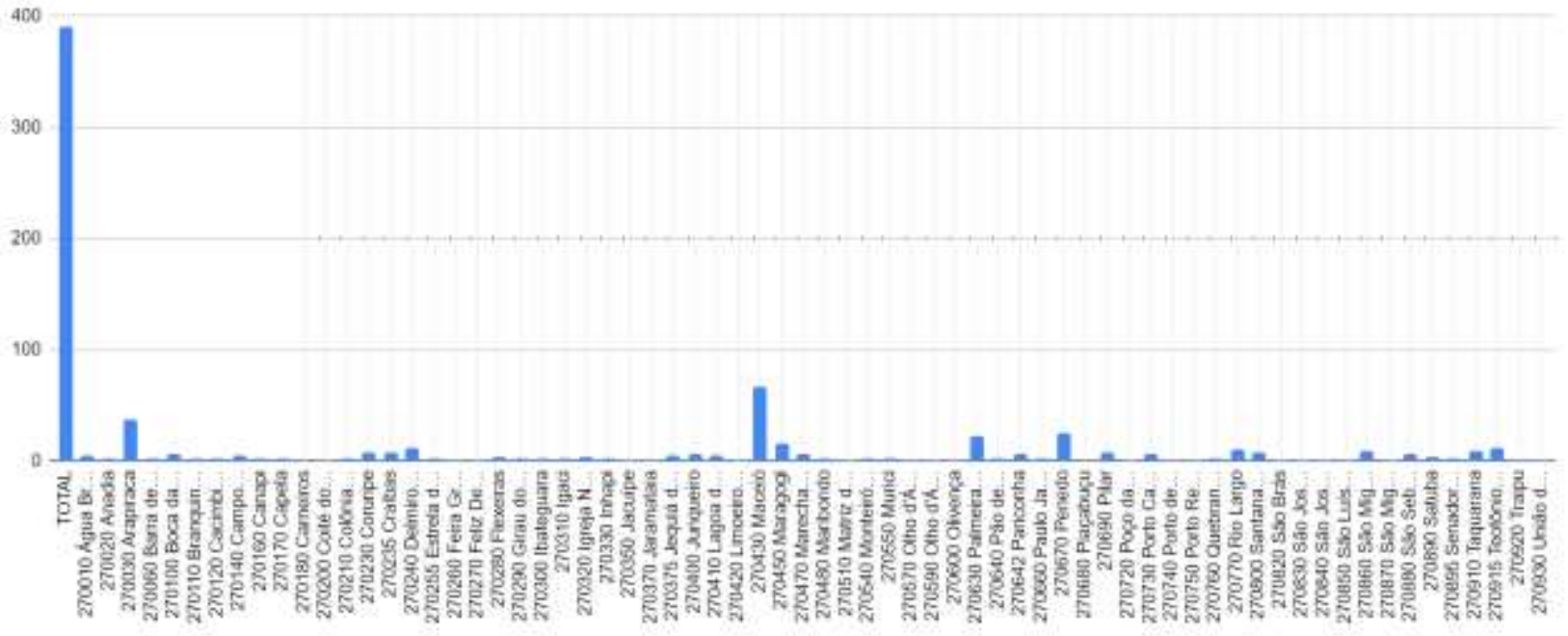

Fonte: Dados da pesquisa (2021) 
Na Estado de Alagoas, entre os anos de 2002 e 2012, o município de Maceió liderou o número de amputações em pé diabético por Diabetes Mellitus de acordo com o cadastro do programa HIPERDIA foram 68,48\% em pessoas do sexo feminino (Gráfico 10).

Gráfico 10 - Amputações por Diabetes Mellitus em Maceió - Alagoas por sexo de acordo com o cadastro do programa HIPERDIA no período de 2002 a 2012

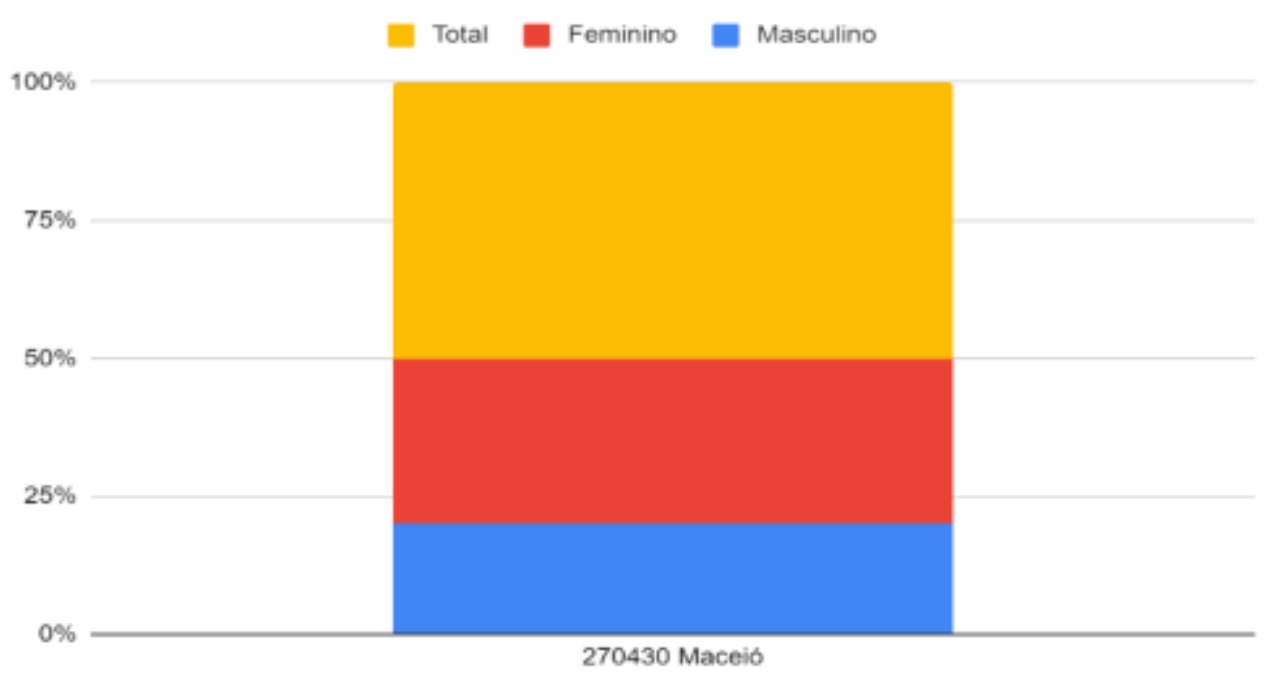

Fonte: Dados da pesquisa (2021)

Na Estado de Alagoas, entre os anos de 2002 e 2012, o município de Maceió liderou o número de amputações em pé diabético por Diabetes Mellitus de acordo com o cadastro do programa HIPERDIA foram respectivamente 17,76\% acometidas em pessoas na faixa etária entre 60 a 64 anos, 15,89\% entre 55 a 59 anos e 14,95\% entre 65 a 69 anos (Gráfico 11).

Gráfico 11 - Amputações por Diabetes Mellitus em Maceió - Alagoas por faixa etária de acordo com o cadastro do programa HIPERDIA no período de 2002 a 2012.

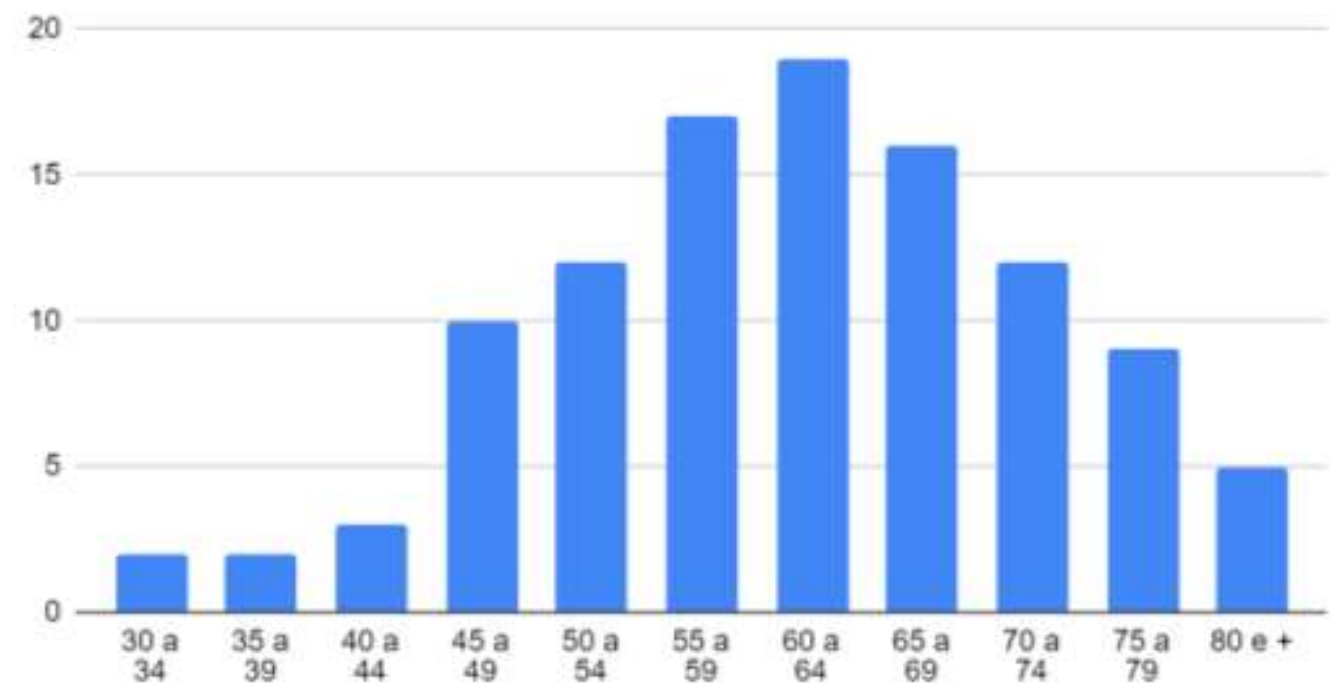

Fonte: Dados da pesquisa (2021) 
Na região Norte, entre os anos de 2010 e 2020, Pará, Roraima e Tocantins lideraram as prevalências de amputações de MMII para 100.000 habitantes, e Pará o estado que apresentou um crescimento potencial nos anos de 2010 a 2019. A queda da prevalência em cada estado no ano de 2020 se deu pelo período de coleta de dados, pois o Sistema de Informações Hospitalares do SUS apenas consta dados até Agosto de 2020 (Gráfico 12).

Gráfico 12 - Amputações por 100.000 habitantes nos estados do Norte entre 2010 e 2020.

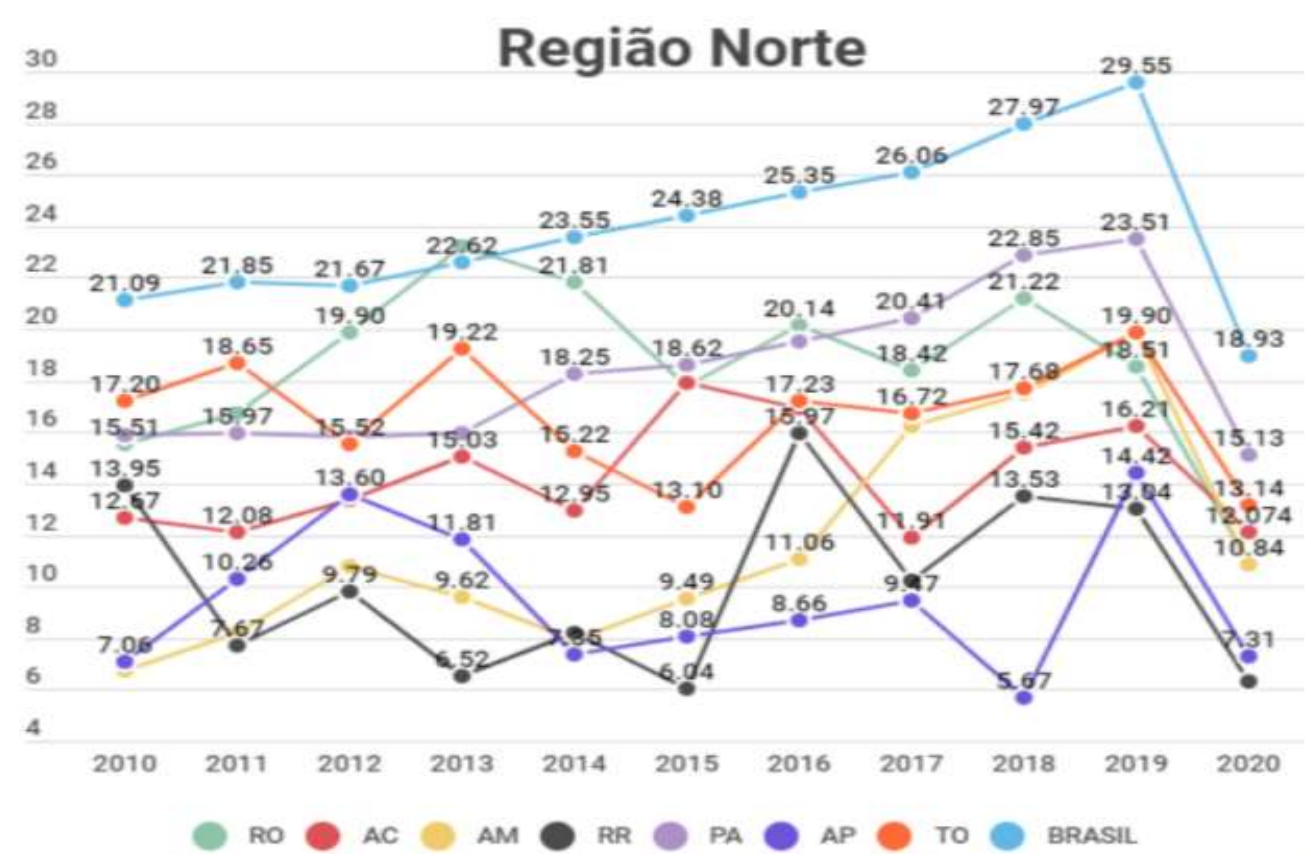

Fonte: Dados da pesquisa (2021)

Na região Sul, entre os anos de 2010 e 2020, Rio Grande do Sul lidera as prevalências de amputações de MMII para 100.000 habitantes em relação ao Brasil, e Santa Catarina foi o estado que apresentou um crescimento potencial nos anos de 2015 a 2019. A queda da prevalência em cada estado no ano de 2020 se deu pelo período de coleta de dados, pois o Sistema de Informações Hospitalares do SUS apenas consta dados até Agosto de 2020 (Gráfico 13).

Gráfico 13 - Amputações por 100.000 habitantes nos estados do Sul entre 2010 e 2020.

\section{Região Sul}

38

36

34

32

30

28

26

24

22

20

$1 \mathrm{n}$

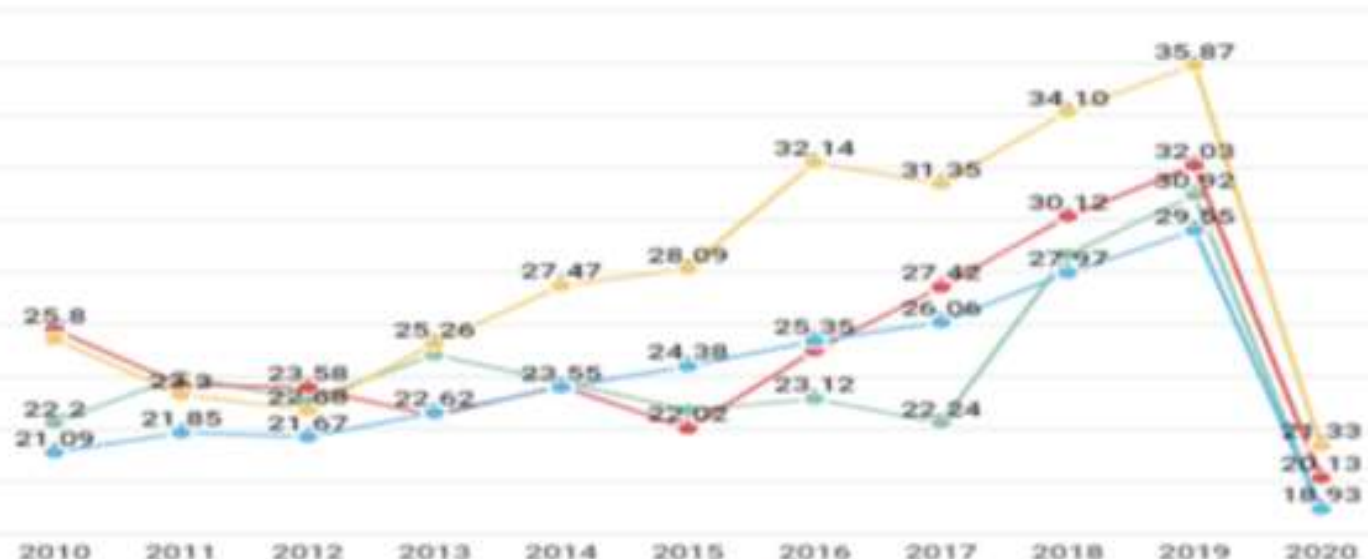

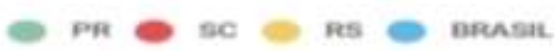

Fonte: Dados da pesquisa (2021) 
Na região Sudeste, entre os anos de 2010 e 2020, Espirito Santo lidera as prevalências de amputações de MMII para 100.000 habitantes em relação ao Brasil, e Rio de Janeiro foi o estado que apresentou um crescimento potencial nos anos de 2014 a 2019. A queda da prevalência em cada estado no ano de 2020 se deu pelo período de coleta de dados, pois o Sistema de Informações Hospitalares do SUS apenas consta dados até Agosto de 2020 (Gráfico 14).

Gráfico 14 - Amputações por 100.000 habitantes nos estados do Sudeste entre 2010 e 2020.

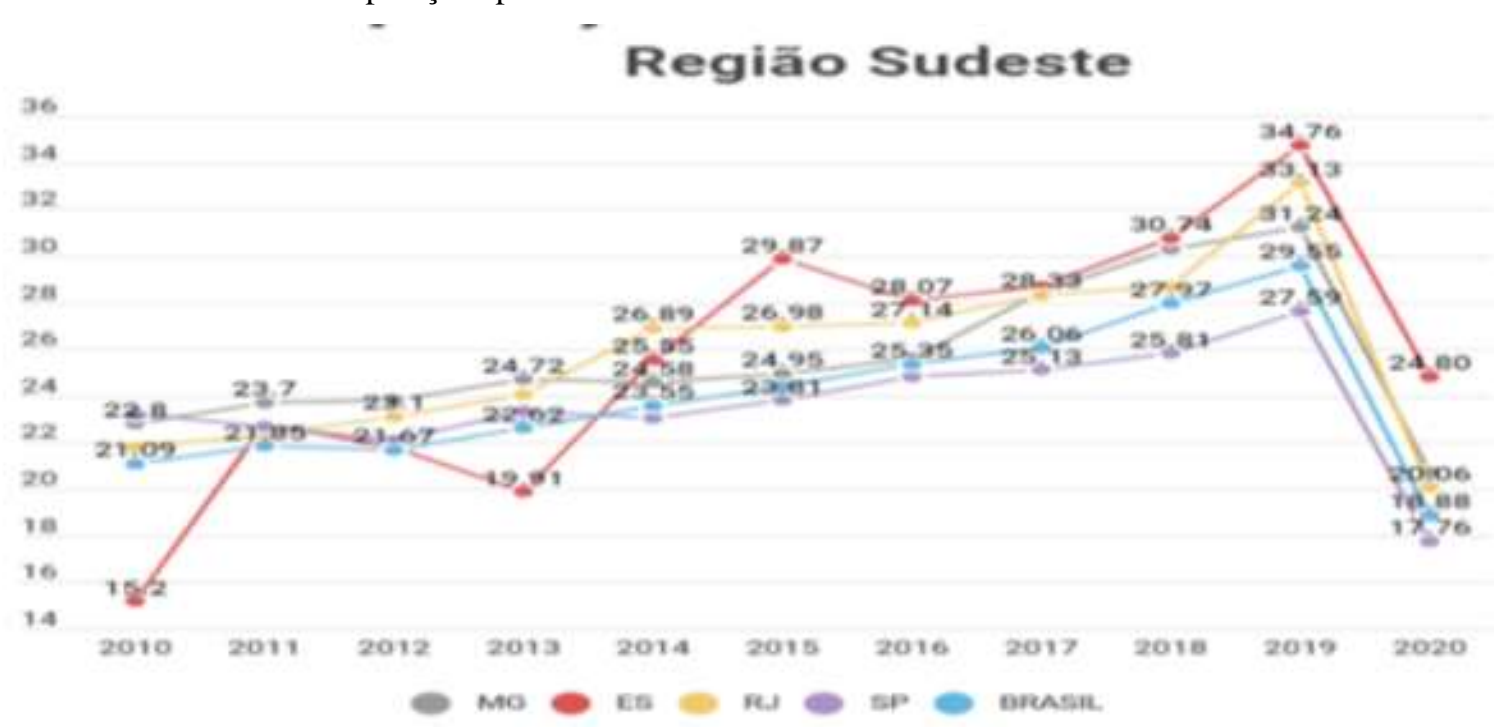

Fonte: Dados da pesquisa (2021)

Na região Centro Oeste, entre os anos de 2010 e 2020, Mato Grosso do Sul lidera as prevalências de amputações de MMII para 100.000, e o Distrito Federal foi o estado que apresentou um crescimento potencial nos anos de 2014 a 2018. A queda da prevalência em cada estado no ano de 2020 se deu pelo período de coleta de dados, pois o Sistema de Informações Hospitalares do SUS apenas consta dados até Agosto de 2020 (Gráfico 15).

Gráfico 15 - Amputações por 100.000 habitantes nos estados do Centro Oeste entre 2010 e 2020.

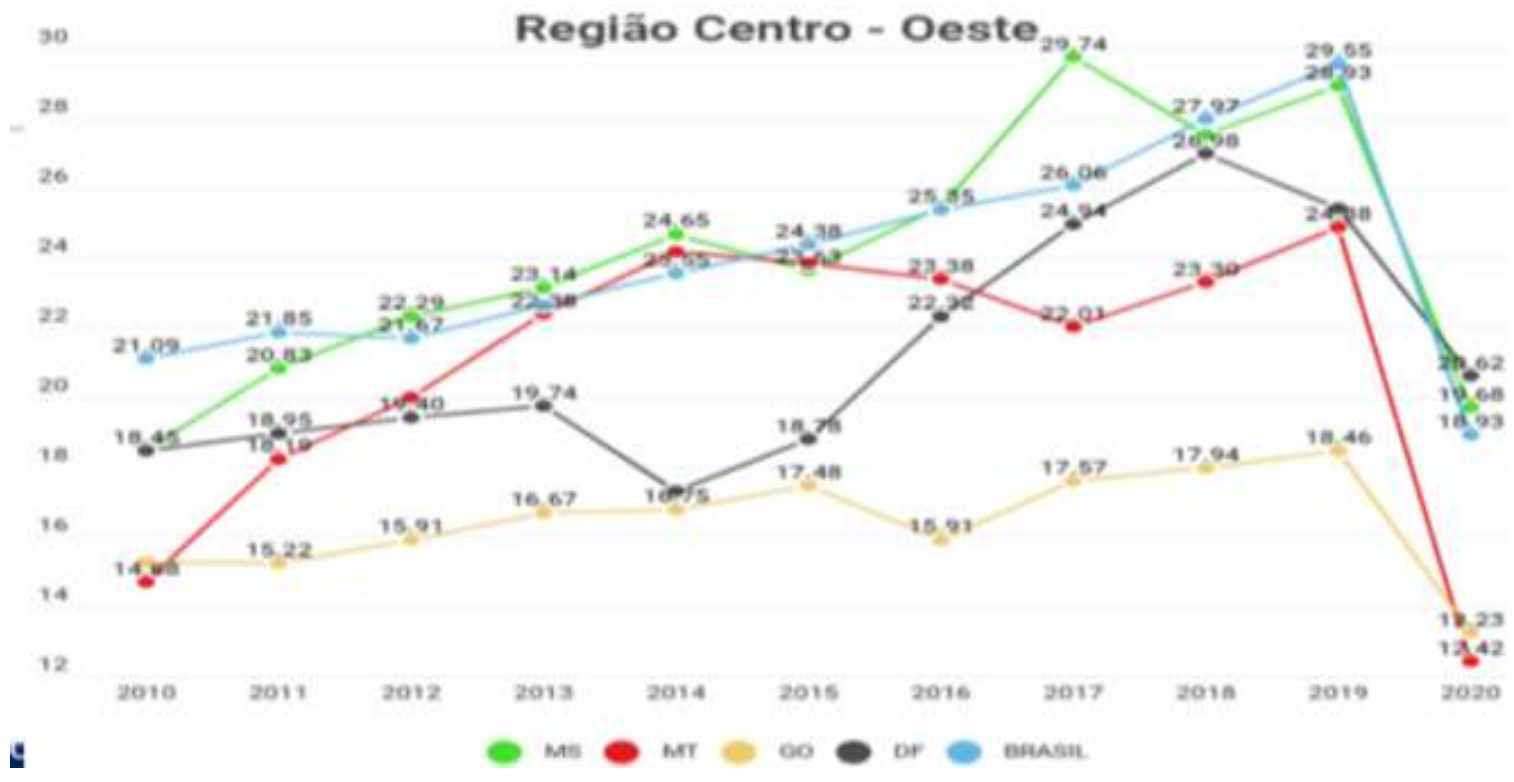

Fonte: Dados da pesquisa (2021) 


\section{Discussão}

A presente pesquisa fornece uma perspectiva epidemiológica atualizada sobre a amputação por Diabetes Mellitus nos estados e regiões do Brasil. De acordo com Peng et al., (2021) o pé diabético é um grave problema de saúde pública mundial, esses pacientes têm comorbidades como: hipertensão, retinopatia diabética e história de tabagismo.

Em uma meta-análise Stern et al., (2017), foram incluídos 16 artigos, com aproximadamente 194.025 participantes de pelo menos 44 países, sendo relatado a amputação por DM de forma substancial. Corroborando com os dados da pesquisa que apresenta elevada morbidade e mortalidade nos pacientes diabéticos.

Sen et al., (2017), em uma meta-análise analisou os fatores de risco para amputação em infecções do pé diabético em 1873 pacientes submetidos à amputação, sendo associado ao tabagismo 1,38 vezes ao risco de amputação de extremidade inferior. Os preditores de amputação foram amputação prévia, osteomielite prévia, Doença Arterial Periférica e retinopatia.

Em um estudo de Coorte Rezende et al., (2009), verificou que 109 diabéticos foram internatos principalmente por apresentar úlceras nos pés, eram diabéticos tipo 2, a equipe multiprofissional não tinha treinamento específico no cuidado do pé diabético, além falta de acesso a reabilitação, foram atendidos exclusivamente pelo sistema público de saúde brasileiro com altos custos e maus resultados.

Bandyk, D.F., (2018), recomenda um tratamento multidisciplinar para o paciente com pé diabético, incluindo consultas anuais na atenção primária à saúde e encaminhamento a um podólogo e cirurgião vascular.

Brocco et al., (2018), relata que a neuroisquemia e infecções podem piorar $85 \%$ as amputações em diabéticos e elas são precedidas de úlcera no pé, gangrena grave ou infecção. Exigem uma abordagem multidisciplinar para orientar os tratamentos, como para a prevenção das amputações e a funcionalidade do pé.

Segundo Grennan, D. (2019), o Diabetes Mellitus podem danificar os vasos sanguíneos de todo o corpo, inclusive dos nervos nas pernas, os pacientes tendem a apresentar a seguinte sintomatologia: dor em queimação ou dormência nos pés (neuropatia periférica) e redução da sensação de dor. Esses fatores predispõem ao surgimento de: calosidades, bolhas, cortes, queimaduras e unhas encravadas, que causam úlceras nos pés diabéticos. A Doença Arterial Periférica que compromete os vasos sanguíneos maiores nas pernas e a hiperglicemia podem causar a demora no tempo de cicatrização.

Grennan, D. (2019), sinaliza sobre as infecções nas úlceras dos pés diabéticos, o osso subjacente a uma úlcera pode infeccionar se a úlcera for profunda. A infecção óssea é chamada de osteomielite e pode causar a morte do osso. E esse é geralmente removido por amputação da parte afetada do pé ou perna.

A American Diabetes Association - Diabetes Care (2019) recomenda: Que todos pacientes devem ser avaliados para neuropatia periférica diabética começando no diagnóstico de diabetes tipo 2 e 5 anos após o diagnóstico de diabetes tipo 1 e pelo menos anualmente a partir de então. Além de avaliação para polineuropatia simétrica distal, com avaliação de sensibilidade usando diapasão de $128 \mathrm{~Hz}$ monofilamento de $10 \mathrm{~g}$ para identificar os pés em risco de ulceração e amputação. E deve ser otimizado tratamento precoce para controle da glicose de modo a prevenir ou atrasar o desenvolvimento de neuropatia em pacientes com diabetes tipo 1 e para retardar a progressão da neuropatia em pacientes com diabetes tipo 2 .

Rorive, M., \& Scheen, A. J. (2019), corrobora com dados dessa pesquisa sobre o cuidado através de equipe multidisciplinar e orienta o rastreio diário do pé diabético como melhor opção para prevenção.

Segundo Bém, R., et al., (2020), o pé diabético está associado à alta morbidade e mortalidade, levando à amputação de membros inferiores. O tratamento desses pacientes possuem um elevado custo, incluindo descarga do pé afetado com sandálias apropriadas, tratamento da infecção e revascularização.

O acompanhamento e a aplicação de abordagens preventivas, como o uso de calçados adequados e cuidados com os pés, podem reduzir a incidência de ulcerações em até 50\%. As limitações da pesquisa foram: os dados não especificaram as amputações /desarticulações de membros inferiores em traumáticas e não traumáticas. Os resultados correspondem a 
informações cadastradas no DATASUS/ TABNET, podendo ter viés de não cadastramento pelos Estados e Municípios. Os dados apresentados apresentam limitações pelo fato dos dados relacionados a amputação por Diabetes Mellitus só estarem disponíveis pelo TABNET quando cadastrados pelo programa HIPERDIA no período de 2002 a 2013.

A principal implicação para futuras pesquisas é que devem ser relatadas associações de amputações não traumáticas e traumáticas por Diabetes Mellitus e o tipo de DM I ou II. Como também a descrição do quantitativo de pacientes com pé diabético, especificando a localização da lesão (dedo, parte do pé, pé completo e/ou perna). E as comorbidades associadas e história prévia de ulceração e/ou amputação. Além da relevância do registro e atualização dos dados do DATASUS/TABNET.

A implicação para a prática clínica da presente pesquisa é que o paciente com DM deve sempre ser incluído no exame físico avaliação dos pés e pernas de modo a prescrever orientações e tratamento precoce, a fim de evitar infecções, ulcerações, amputações e óbito. Além do elevado custo aos cofres públicos e privados.

É recomendável realizar o rastreamento precoce dos pacientes DM através de exames laboratoriais como: hemoglobina glicosilada A1c (HbA1c), glóbulos brancos (WBC), albumina (ALB), ácido úrico no sangue (BUA) e fibrinogênio, exames de imagem como ultrassonografia de membros inferiores Peng et al., (2021).

\section{Conclusão}

Nossos resultados indicam que as regiões sudeste e nordeste têm elevada prevalência de internação hospitalar, amputação e mortalidade por Diabetes Mellitus. Esses dados demonstram a necessidade de realização de assistência precoce a essa população a fim de evitar morbidade e mortalidade. Além de corroborar como ferramenta para os gestores para implantação de estratégias terapêuticas eficaz.

\section{Agradecimentos}

A Coordenação de Aperfeiçoamento de Pessoal de Nível Superior (CAPES).

\section{Referências}

American Diabetes Association. (2019). Complicações microvasculares e cuidados com os pés: padrões de cuidados médicos em diabetes - 2019. Diabetes Care, 42 (1), S124-S138.

Assumpção, E. C, Pitta, G. B. B, Macedo A. C. L, Mendonça G. B, Albuquerque, L. C. A, Lyra, L. C. B., Timbó, R. M., \& Buarque, T. L. L. (2009). Comparação dos fatores de risco para amputações maiores e menores em pacientes diabéticos de um Programa de Saúde da Família. Jornal Vascular Brasileiro, 8 (2),133138.

Atlas, I. D. (2013). Online version of IDF Diabetes Atlas: http://www. idf. org/diabetesatlas. International Diabetes Federation.

Bandyk, D.F., (2018). The diabetic foot: Pathophysiology, evaluation, and treatment. Semin Vasc Surg. 31(2-4):43-48. 10.1053/j.semvascsurg.2019.02.001. Epub 2019 Feb 6. PMID: 30876640.

Bém, R., Dubský, M., Fejfarová, V., Husáková, J., \& Wosková, V. (2020). Pé diabético. Diabetická noha. Vnitrni lekarstvi, 66 (2), $92-97$.

Boulton, A. J., Vileikyte, L., Ragnarson-Tennvall, G., \& Apelqvist, J. (2005). The global burden of diabetic foot disease. The Lancet, 366 (9498), 1719-1724.

Brocco, E., Ninkovic, S., Marin, M., Whisstock, C., Bruseghin, M., Boschetti, G., \& Volpe, A. (2018). Diabetic foot management: multidisciplinary approach for advanced lesion rescue. The Journal of cardiovascular surgery, 59(5), 670-684.

DATASUS - Sistema de Informações Hospitalares (SIH/SUS). (2021). http://tabnet.datasus.gov.br/cgi/tabcgi.exe?sih/cnv/qiuf.def>.

Grennan, D. (2019). Diabetic foot ulcers. Jama, 321(1), 114-114.

International Diabetes Federation. (2021). https://idf.org/

Geiss, L. S., Li, Y., Hora, I., Albright, A., Rolka, D., \& Gregg, E. W. (2019). Resurgence of diabetes-related nontraumatic lower-extremity amputation in the young and middle-aged adult US population. Diabetes Care, 42 (1), 50-54.

Miyajima, S., Shirai, A., Yamamoto, S., Okada, N., \& Matsushita, T. (2006). Risk factors for major limb amputations in diabetic foot gangrene patients. Diabetes research and clinical practice, 71(3), 272-279. 
Research, Society and Development, v. 10, n. 4, e11910413837, 2021

(CC BY 4.0) | ISSN 2525-3409 | DOI: http://dx.doi.org/10.33448/rsd-v10i4.13837

Nunes, M. A. P., Resende, K. F., Castro, A. A., Pitta, G. B. B., Figueiredo, L. F. P. D., \& Miranda Jr, F. (2006). Fatores predisponentes para amputação de membro inferior em pacientes diabéticos internados com pés ulcerados no estado de Sergipe. Jornal Vascular Brasileiro, 5(2), 123-130.

Schaper, N. C. (2012). Lessons from eurodiale. Diabetes/metabolism research and reviews, 28, 21-26.

Sen, P., Demirdal, T., \& Emir, B. (2019). Meta-analysis of risk factors for amputation in diabetic foot infections. Diabetes/metabolism research and reviews, 35(7), e3165.

Pecoraro, R. E., Reiber, G. E., \& Burgess, E. M. (1990). Pathways to diabetic limb amputation: basis for prevention. Diabetes care, 13(5), 513-521.

Peng, B., Min, R., Liao, Y., \& Yu, A. (2021). Development of Predictive Nomograms for Clinical Use to Quantify the Risk of Amputation in Patients with Diabetic Foot Ulcer. Journal of Diabetes Research, 2021.

Pereira, A. S. et al. (2018). Metodologia da pesquisa científica. UFSM. https://repositorio.ufsm.br/bitstream/handle/1/15824/Lic_Computacao_MetodologiaPesquisa-Cientifica.pdf?sequence $=1$.

Pitta, G. B. B., Castro, A. A., Soares, A. M. M. N., Maciel, C. D. J. J., da Silva, J. D. M., Muniz, V. M. T., \& Asmar, S. B. (2019). Perfil dos pacientes portadores de pé diabético atendidos no Hospital Escola José Carneiro e na Unidade de Emergência Armando Lages. Jornal Vascular Brasileiro, 4(1), 5-10.

Rezende, K. F., Ferraz, M. B., Malerbi, D. A., Melo, N. H., Nunes, M. P., Pedrosa, H. C., \& Chacra, A. R. (2009). Direct costs and outcomes for in patients with diabetes mellitus and foot ulcers in a developing country: The experience of the public health system of Brazil. Diabetes \& Metabolic Syndrome: Clinical Research \& Reviews, 3(4), 228-232.

Rorive, M., \& Scheen, A. J. (2019). Actualités dans la prise en charge du pied diabétique [News in the management of diabetic foot]. Revue medicale suisse, 15(659), 1448-1452.

Santos, V. P. D., Silveira, D. R. D., \& Caffaro, R. A. (2006). Risk factors for primary major amputation in diabetic patients. Sao Paulo Medical Journal, 124(2), 66-70.

Stern, J. R., Wong, C. K., Yerovinkina, M., Spindler, S. J., See, A. S., Panjaki, S., \& Nowygrod, R. (2017). A meta-analysis of long-term mortality and associated risk factors following lower extremity amputation. Annals of vascular surgery, 42, 322-327.

Wrobel, J. S., Mayfield, J. A., \& Reiber, G. E. (2001). Geographic variation of lower-extremity major amputation in individuals with and without diabetes in the Medicare population. Diabetes care, 24(5), 860-864. 